\title{
Cholecystitis after Placement of Covered Self-Expandable Metallic Stents in Patients with Distal Malignant Biliary Obstructions
}

\author{
Masafumi Watanabe ${ }^{1}, K^{\prime}$ osuke Okuwaki ${ }^{1}$, Jun Woo ${ }^{2}$, Mitsuhiro Kida ${ }^{1}$, Hiroshi Imaizumi', Tomohisa Iwai', Hiroshi Yamauchi', Toru \\ Kaneko', Rikiya Hasegawa', Takahiro Kurosu', Naoki Minato', Hiroki Haradome ${ }^{3}$ and Wasaburo Koizumi ${ }^{1}$
}

Department of ${ }^{1}$ Gastroenterology, ${ }^{2}$ Diagnostic Radiology, ${ }^{3}$ Radiological Advanced Medicine, Kitasato University School of Medicine, Sagamihara, Kanagawa, Japan

Background/Aims: Cholecystitis can occur after the placement of covered self-expandable metallic stents for distal malignant biliary obstructions. We aimed to identify risk factors for cholecystitis following covered self-expandable metallic stent placement.

Methods: We investigated risk factors related to cholecystitis following covered self-expandable metallic stent placement in 118 patients with distal malignant biliary obstructions between January 1, 2015 and April 30, 2019. Endoscopic assessments and tumor invasion to the arteries feeding the gallbladder were determined by a pancreaticobiliary endoscopist and a radiologist, respectively. Results: The median patient age was 72 years (men, $61.0 \%$ ). The flow of the contrast agent into the gallbladder and tumor involvement in the orifice of the cystic duct were observed in $35(29.7 \%)$ and $35(29.7 \%)$ patients, respectively. During the observation period (median, 179 days), cholecystitis occurred in $18(15.3 \%)$ patients. Multivariate analysis revealed the flow of the contrast agent into the gallbladder $(p=0.023)$ and tumor involvement in the orifice of the cystic duct $(p=0.005)$ as significant independent risk factors associated with cholecystitis.

Conclusions: The flow of the contrast agent into the gallbladder and tumor involvement in the orifice of the cystic duct are potential independent risk factors for cholecystitis following the placement of covered self-expandable metallic stents. A follow-up prospective study is warranted to validate their influence. Clin Endosc 2021;54:589-595

Key Words: Cholecystitis; Endoscopic retrograde cholangiopancreatography; Malignant biliary obstruction; Risk factors; Selfexpandable metallic stents

\section{INTRODUCTION}

Since its introduction in the 1980s, endoscopic stent placement has been used to treat patients with jaundice associated with distal malignant biliary obstructions that occur in cases of pancreatic and biliary tract cancer and to help maintain an effective quality of life. ${ }^{1-4} \mathrm{~A}$ longer time to recurrent biliary ob-

Received: May 21, 2020 Revised: June 29, 2020

Accepted: July 20, 2020

Correspondence: Masafumi Watanabe

Department of Gastroenterology, Kitasato University School of Medicine, 1-15-1 Kitasato, Minami, Sagamihara, Kanagawa 252-0374, Japan

Tel: +81-42-778-8111, Fax: +81-42-778-8390, E-mail: m.wata@kitasato-u.ac.jp

ORCID: https://orcid.org/0000-0003-1278-9021

(c) This is an Open Access article distributed under the terms of the Creative Commons Attribution Non-Commercial License (http://creativecommons.org/ licenses/by-nc/3.0) which permits unrestricted non-commercial use, distribution, and reproduction in any medium, provided the original work is properly cited. struction (TRBO), lower rates of complications, and a reduced need for re-interventions with a self-expandable metallic stent (SEMS) compared to a plastic stent (PS) have been reported in recent meta-analyses. ${ }^{5}$ A randomized controlled trial ${ }^{6}$ and a meta-analysis ${ }^{7}$ comparing covered and uncovered SEMS (CSEMS, USEMS) have also shown the superior TRBO of CSEMSs compared to USEMSs, which explains why CSEMSs have been used more frequently in recent times. However, a $3.5 \%-12 \%$ incidence of postoperative cholecystitis has been reported in patients with CSEMS placement. ${ }^{8-12}$ Cholecystitis causes abdominal pain and delays the time to scheduled chemotherapy, which negatively affects patient therapeutic strategies, and consequently, their vital prognosis. Therefore, it is imperative to identify the risk factors for cholecystitis associated with CSEMS placement.

Some of the previously reported risk factors for cholecystitis following CSEMS placement are tumor involvement in the 
orifice of cystic duct (OCD) ${ }^{8-13}$ high axial force (AF) SEMS, ${ }^{10}$ SEMS length $\leq 60 \mathrm{~mm},{ }^{10}$ and tumor invasion to the arteries feeding the gallbladder. ${ }^{8}$ However, the retrospective nature of these studies and the use of gastroenterologists to assess tumor invasion to the arteries feeding the gallbladder are weaknesses of these works. Therefore, in this retrospective study, radiologists who had no access to patient data independently determined the presence of tumor invasion to the arteries feeding the gallbladder to investigate the risk factors for cholecystitis development following CSEMS placement.

\section{MATERIALS AND METHODS}

\section{Study design}

We performed a retrospective study using endoscopic retrograde cholangiopancreatography (ERCP) registry data of Kitasato University Hospital. The study adhered to the principles of the Declaration of Helsinki. Our study was reviewed and approved by our institutional ethics committee (approval number: B18-113). The study is registered with the University Hospital Medical Information Network (UMIN) Center of Japan (Clinical trial registration number: UMIN000040028).

\section{Patients}

Our analysis included 160 consecutive patients who underwent SEMS placement for distal malignant biliary obstructions at the Kitasato University Hospital between January 1, 2015, and April 30, 2019. Informed consent was obtained from all individual participants included in the study. Patients who had a history of cholecystectomy, could not be followed-up after SEMS placement due to transfer to other hospitals, underwent USEMS and preparatory or concurrent gallbladder drainage placement, and were not assessed by multidetector computed tomography (MDCT) before SEMS placement were excluded from the analysis.

\section{Procedures}

The following parameters were investigated in the present study: age, sex, primary disease, previous biliary duct drainage using PS before SEMS placement, pre-SEMS papillary treatment, flow of contrast agent into the gallbladder (including the cystic duct) during the SEMS placement, type of SEMS used (SEMS length and braided or laser-cut type), location of the placed SEMS (above or across the papilla), tumor involvement to the OCD, tumor invasion to the arteries feeding the gallbladder, gallstones presence, TRBO and survival time during the observation period, and incidence of cholecystitis. Medical data for the period from SEMS placement to the last day of observation (August 31, 2019) were collected from electronic charts.

The flow of the contrast agent to the gallbladder was determined as positive when it was visible on fluoroscopic images (Fig. 1). Consensus was reached through a discussion between two Board Certified Trainers of the Japan Gastroenterological Endoscopy Society (JGES). Tumor invasion on the artery feeding the gallbladder and its origin artery was determined by MDCT performed before SEMS placement using the method described by Sogabe et al. ${ }^{8}$ by observation of "tumor extension around the artery and irregular shape of the artery by MDCT prior to SEMS placement". The latter was discussed over a discussion between two Board Certified Fellows of the Japan Radiological Society (JRS) who had no access to patient data (Fig. 2). OCD involvement was defined when the tumor extended around the OCD on MDCT/magnetic resonance cholangiopancreatography (MRCP)/intraductal ultrasonography (IDUS) or when the cystic duct was derived from irregular biliary narrowing using endoscopic retrograde cholangiography (ERC). MDCT and MRCP were assessed by two JRS Board Certified Fellows who had no access to patient data. Consensus was reached through discussion. Assessments of ERC and IDUS results were made by two JGES Board Certified Trainers. Positive findings in any of the aforementioned modalities were indicative of tumor involvement to the OCD (Fig. 3). The evaluation of OCD involvement by IDUS and ERC was assessed during the same test session immediately before SEMS placement. Definitions and severity grading of cholecystitis conformed to the TOKYO criteria 2014 for transpapillary biliary stenting. ${ }^{14}$



Fig. 1. Flow of contrast agent into the gallbladder. Flow of the contrast agent into the gallbladder was deemed positive when the contrast agent was observed flowing into the gallbladder or cystic duct on fluoroscopic imaging in endoscopic retrograde cholangiopancreatography. 




Fig. 2. Tumor invasion to the cystic artery. Tumor invasion to the cystic artery (arrow) was visible on multidetector computed tomography.

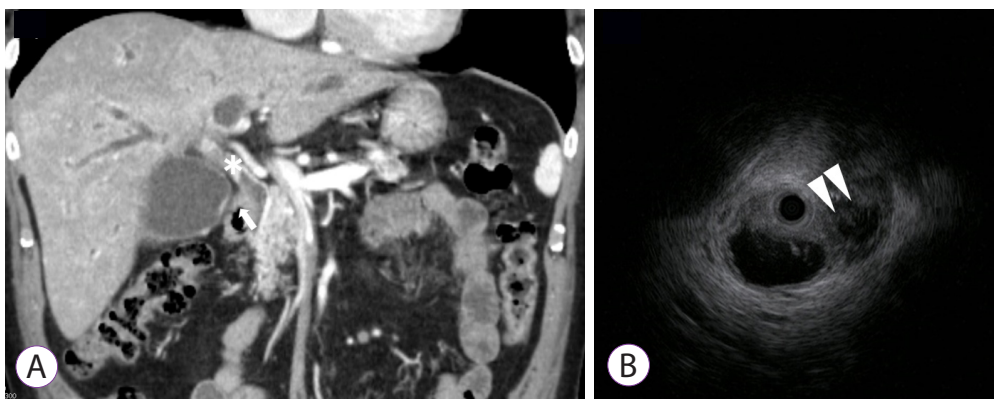

Fig. 3. Tumor involvement to the orifice of cystic duct. Multidetector computed tomography (A) shows cholangiocarcinoma of the common bile duct $(*)$ and contrast enhancement at the cystic duct (arrow). Infiltration of the tumor from the bile to the cystic duct was visible on intraductal ultrasonography (arrowheads) (B).

\section{Stent insertion}

The operating physician determined whether a papillary treatment was performed before SEMS placement. SEMS length and type were selected by the operating physician to achieve adequate coverage of the stricture as imaged on ERC. The CSEMSs used in this study were the BONA STENT ${ }^{\circledR}$ (Sewoon Medical Co., Ltd., Seoul, Korea), Evolution ${ }^{\circledR}$ (COOK Medical, Bloomington, IN, USA), M-intraductal stent ${ }^{\circledR}$ (SciTech Inc., Seoul, Korea), HANARO STENT ${ }^{\circledR}$ (MI-Tech, Seoul, Korea), X-suit NIR ${ }^{\circledR}$ (Olympus Medical Systems, Tokyo, Japan), and WallFlex ${ }^{\circledast}$ (Boston Scientific Co., Natick, MA, USA).

\section{Statistical analyses}

TRBO was estimated using the Kaplan-Meier method and was compared between the groups using the log-rank test. A logistic regression model was used for univariate and multivariate analyses of independent factors for cholecystitis. All factors with $p<0.10$ in the univariate analysis were further assessed using multivariate analysis. Statistical analyses were performed using SPSS Base 17.0 (SPSS Inc., Chicago, IL, USA) and $\mathrm{R}$ version 3.2.4 statistical packages ( $\mathrm{R}$ Foundation for Statistical Computing, Vienna, Austria). The statistically significant level was set at $p<0.05$.

\section{RESULTS}

\section{Patients}

Of the 160 consecutive patients, $17,9,8,4$, and 4 patients who had cholecystectomy history, could not be followed-up, underwent USEMS placement, had previous or concurrent gallbladder drainage for SEMS placement, and were not assessed by MDCT before SEMS placement, respectively, were excluded. Thus, 118 patients (median age, 72 years [range,
22-92 years]; sex, 72 men [61.0\%] and 46 women [39.0\%]) were finally enrolled. Table 1 shows the patients' background characteristics. Flow of the contrast agent in the gallbladder was observed in 35/118 (29.7\%) patients, and tumor involvement at the OCD was also observed in the same number of patients. In $12 / 118$ patients (10.2\%), both contrast agent in the gallbladder and tumor involvement at the OCD were observed. The direct gall bladder-feeding artery was identified as the cystic artery in 114/118 (96.6\%) patients and was indistinguishable in four (3.4\%) patients. The most common origin of the cystic artery was the right hepatic artery in 95/118 (80.5\%) patients. The tumor invaded the cystic artery and its origin in $3 / 118(2.5 \%)$ and 10/118 (8.5\%) patients, respectively. The tumor had invaded both of the cystic artery and its origin in one $(0.8 \%)$ patient.

\section{Incidence of cholecystitis}

During the observation period (median, 179 days [range, 17-1,231 days]), cholecystitis occurred in 18 (15.3\%) patients after a median of 13 days (range, 1-269 days) following CSEMS placement. Thirteen patients developed early cholecystitis (72.2\%) within 30 days of CSEMS placement.

The cholecystitis was mild, moderate, and severe in 1 (5.6\%), $16(88.9 \%)$, and 1 (5.6\%) patients, respectively. The first treatment performed for cholecystitis was percutaneous transhepatic gallbladder aspiration (PTGBA) only in seven patients, PTGBA plus CSEMS removal in four, percutaneous transhepatic gallbladder drainage (PTGBD) only in four, watchful waiting with antibiotic therapy in two, and CSEMS removal plus endoscopic nasogallbladder drainage in one patient (Table 2). Four patients who underwent PTGBA experienced only recurrent cholecystitis. However, all cases of recurrent cholecystitis were treated with PTGBA, PTGBD, CSEMS removal, or cholecystectomy. 
Table 1. Patient Characteristics $(n=118)$

\begin{tabular}{|c|c|}
\hline & $n(\%)$ \\
\hline Median age, yr [range] & $72[22-92]$ \\
\hline Sex, male/female & $72(61.0) / 46(39.0)$ \\
\hline \multicolumn{2}{|l|}{ Etiology of malignant biliary obstruction } \\
\hline Pancreatic cancer & $98(83.1)$ \\
\hline Biliary tract cancer & $15(12.7)$ \\
\hline Other malignancies & $5(4.2)$ \\
\hline Previous biliary duct drainage using plastic stent & $78(66.1)$ \\
\hline \multicolumn{2}{|l|}{ Papillary treatment } \\
\hline EST & $104(88.1)$ \\
\hline $\mathrm{EPBD}$ & $2(1.7)$ \\
\hline None & $12(10.2)$ \\
\hline Flow of contrast agent into gallbladder & $35(29.7)$ \\
\hline \multicolumn{2}{|l|}{ Median stent length } \\
\hline$>60 \mathrm{~mm} / \leq 60 \mathrm{~mm}$ & $65(55.1) / 53(44.9)$ \\
\hline \multicolumn{2}{|l|}{ Location of the SEMS placement } \\
\hline Across/above the papilla & $107(90.7) / 11(9.3)$ \\
\hline Tumor involvement to OCD & $35(29.7)$ \\
\hline \multicolumn{2}{|l|}{ Artery feeding the gallbladder } \\
\hline Cystic artery & $114(96.6)$ \\
\hline Unidentifiable & $4(3.4)$ \\
\hline \multicolumn{2}{|l|}{ Origin of the cystic artery } \\
\hline RHA & $95(80.5)$ \\
\hline Replaced RHA & $10(8.5)$ \\
\hline GDA & $4(3.4)$ \\
\hline PHA & $2(1.7)$ \\
\hline LHA & $1(0.8)$ \\
\hline SMA & $1(0.8)$ \\
\hline Unidentifiable & $5(4.2)$ \\
\hline Tumor invasion to the cystic artery & $3(2.5)$ \\
\hline Tumor invasion to the origin of the cystic artery & $10(8.5)$ \\
\hline Gallbladder stones & $17(14.4)$ \\
\hline
\end{tabular}

EPBD, endoscopic papillary balloon dilation; EST, endoscopic sphincterotomy; GDA, gastroduodenal artery; LHA, left hepatic artery; OCD, orifice of cystic duct; PHA, proper hepatic artery; RHA, right hepatic artery; SEMS, self-expandable metallic stent; SMA, superior mesenteric artery.
Table 2. Incidence of Cholecystitis $(n=18)$

\begin{tabular}{lc}
\hline \multicolumn{2}{l}{ Severity grading of cholecystitis after stent placement } \\
Mild & $\boldsymbol{n}(\mathbf{\%})$ \\
Moderate & $16(8.6)$ \\
Severe & $1(5.6)$ \\
Initial therapy for cholecystitis & \\
PTGBA only & $7(38.9)$ \\
PTGBA plus CSEMS removal & $4(22.2)$ \\
PTGBD only & $4(22.2)$ \\
Watchful waiting with antibiotics & $2(11.1)$ \\
CSEMS removal plus ENGBD & $1(5.6)$ \\
\hline CSEMS, covered self-expandable metallic stent; ENGBD, endo- \\
scopic nasogallbladder drainage; PTGBA, percutaneous transhe- \\
patic gallbladder aspiration; PTGBD, percutaneous transhepatic \\
gallbladder drainage.
\end{tabular}

\section{Time to recurrent biliary obstruction of self- expanding metallic stents}

The TRBO was 291 days (range, 0-1,159 days), and there was no significant difference between patients who developed cholecystitis (median, 285 days [range, 7-530 days]) and those who did not (median, 291 days [range, $0-1,159$ days]) $(p=0.797)$. The median overall survival was 260 days (range, $17-1,231$ days), with no significant differences between those who developed cholecystitis (median, 197 days [range, 35-696 days]) and those who did not (median, 260 days [range, 17$1,231$ days $])(p=0.162)$.

\section{Analysis of cholecystitis}

The univariate and multivariate analyses revealed that the contrast agent flow into the gallbladder (univariate: odds ratio [OR], 3.750; 95\% confidence interval [CI], 1.333-10.546; $p=0.012$, multivariate: $\mathrm{OR}, 3.496$; $95 \% \mathrm{CI}, 1.184-10.322$; $p=0.023$ ) and the OCD tumor involvement (univariate: OR, 4.976; 95\% CI, 1.736-14.262; $p=0.003$, multivariate: OR, 4.702; 95\% CI, 1.591-13.895; $p=0.005)$ were significant risk factors associated with cholecystitis (Table 3). In 12/118 patients $(10.2 \%)$, both contrast agent in the gallbladder and tumor involvement at the OCD were observed. Seven of 12 patients (58.3\%) had developed cholecystitis. Of the seven cholecystitis cases, five occurred in the early stage and two occurred in the late stage. 
Table 3. Analysis of Risk Factors Related to Cholecystitis

\begin{tabular}{|c|c|c|c|c|}
\hline & \multicolumn{2}{|c|}{ Univariate analysis } & \multicolumn{2}{|c|}{ Multivariate analysis } \\
\hline & Patients & $p$-value & OR $(95 \% \mathrm{CI})$ & $p$-value \\
\hline Sex & & 0.594 & & \\
\hline Male & $12 / 72$ & & & \\
\hline Female & $6 / 46$ & & & \\
\hline Causative disease & & n.c. & & \\
\hline Pancreatic and biliary cancer & $18 / 113$ & & & \\
\hline Others & $0 / 5$ & & & \\
\hline Previous biliary duct drainage using plastic stent & & 0.553 & & \\
\hline Yes & $13 / 78$ & & & \\
\hline No & $5 / 40$ & & & \\
\hline Flow of contrast agent into gallbladder & & 0.012 & $3.496(1.184-10.322)$ & 0.023 \\
\hline Yes & $10 / 35$ & & & \\
\hline No & $8 / 83$ & & & \\
\hline Median stent length & & 0.287 & & \\
\hline$>60 \mathrm{~mm}$ & $12 / 65$ & & & \\
\hline$\leq 60 \mathrm{~mm}$ & $6 / 53$ & & & \\
\hline Location of SEMS placement & & 0.255 & & \\
\hline Across the papilla & $15 / 107$ & & & \\
\hline Above the papilla & $3 / 11$ & & & \\
\hline Tumor involvement at the OCD & & 0.003 & $4.702(1.591-13.895)$ & 0.005 \\
\hline Yes & $11 / 35$ & & & \\
\hline No & $7 / 83$ & & & \\
\hline Tumor invasion to cystic artery ${ }^{*}$ & & n.c. & & \\
\hline Yes & $0 / 3$ & & & \\
\hline No & $18 / 111$ & & & \\
\hline Tumor invasion to the origin of cystic artery ${ }^{\star \star}$ & & 0.596 & & \\
\hline Yes & $1 / 10$ & & & \\
\hline No & $17 / 103$ & & & \\
\hline Gallbladder stones & & 0.767 & & \\
\hline Yes & $3 / 17$ & & & \\
\hline No & $14 / 101$ & & & \\
\hline Structure of SEMS & & 0.398 & & \\
\hline Braded & $17 / 115$ & & & \\
\hline Laser-cut & $1 / 3$ & & & \\
\hline
\end{tabular}

CI, confidence interval; n.c., not calculated; OCD, orifice of cystic duct; OR, odds ratio; SEMS, self-expandable metallic stent.

${ }^{\star}$ Four patients were excluded because of non-evaluability.

${ }^{*}$ Five patients were excluded because of non-evaluability.

\section{DISCUSSION}

Nakai et al. conducted a multicenter retrospective investigation to identify the risk factors for post-SEMS cholecystitis and reported that OCD tumor involvement (OR, 5.4; $p<0.001)$, SEMS with high AF (OR, 5.33; $p=0.002)$, and SEMS length $\leq 60 \mathrm{~mm}(\mathrm{OR}, 3.19 ; p=0.010)$ were risk factors. ${ }^{10}$ They proposed that the compression of the OCD, which has lost elas- 
ticity due to the tumor invasion by the high AF SEMS, leads to impaired bile efflux, thereby inducing cholecystitis. They explained that an SEMS length $\leq 60 \mathrm{~mm}$ was attributed to a shorter distance from the stent ends to the stricture, which characterizes a short SEMS and increases $\mathrm{AF}^{15}$ We also found that 11 (31.4\%) of 35 patients with tumor involvement to the OCD had cholecystitis, which corroborated with previous studies $^{8-13}$; hence, OCD tumor involvement was identified as a significant risk for cholecystitis onset. These data may suggest that transpapillary biliary drainage (BD) using CSEMS should be avoided in patients in whom OCD tumor involvement has been identified preoperatively.

Isayama et al. reported that endoscopic ultrasound-guided BD (EUS-BD) should be considered for patients in high risk, including those with OCD tumor involvement to prevent cholecystitis after SEMS placement. ${ }^{16}$ With the recent increase in reports of therapeutic outcomes of EUS-BD, new meta-analyses have reported comparable outcomes for ERCP and EUS$\mathrm{BD} .^{17}$ There are two major issues that should be examined in future studies: (1) Whether a route for EUS-BD or the transpapillary approach is beneficial for preventing cholecystitis and (2) whether this route will produce the same TRBO as transpapillary BD with SEMS for cases in which OCD tumor involvement is known before SEMS placement.

In this study, flow of the contrast agent into the gallbladder was also identified as a significant risk factor for cholecystitis. In a recent study evaluating endoscopic biliary stenting, including the use of PSs in patients with malignant extrahepatic bile duct obstruction, Kim et al. previously evaluated contrast agent injection into the gallbladder as an important predictive factor for cholecystitis and suggested that it may potentially be associated with the location of cancer because if the cancer is in the vicinity of the OCD, there may be retention of the contrast agent. ${ }^{18}$ Klimczak et al. made cultures of bile harvested from the initial drainage of patients with jaundice caused by malignant biliary stricture without signs of infection and found that the culture was positive in $56 \%$ of them. ${ }^{19}$ Furthermore, it is known that post-endoscopic sphincterotomy bile is often colonized by enteric bacteria. ${ }^{20}$ Cholangiography is performed to make an accurate assessment of the location and extent of stricture for SEMS placement. We can assume that the contrast agent flow beyond the cystic duct indicates that a substantial amount of bile containing bacteria flows inside the gallbladder during the cholangiography procedure, and that the subsequent SEMS placement triggers bile flow disturbance. Of note, in this study, there were 12 cases of positive cystic duct infiltration and contrast agent inflow into the cystic duct. Partial obstruction of the cystic duct opening was highly likely in these 12 cases, and of these, 7 (58.3\%) exhibited cho- lecystitis. If both factors are positive, this may increase the risk of cholecystitis development. As this might be the mechanism of cholecystitis onset, it may be prudent to avoid injection of large amounts of the contrast agent before SEMS placement, and aspirate and remove the highest possible amount of bile from the biliary duct before cholangiography, as preventive measures.

Tumor invasion to the arteries feeding the gallbladder, which was raised as a significant risk factor of cholecystitis onset by Sogabe et al., ${ }^{8}$ was not identified as a risk factor in the present investigation. This difference may be caused by the rate of tumor invasion to the feeding artery, which was lower than that reported by Sogabe et al. (22.4\%), and the differences in the characteristics of the populations studied. ${ }^{8}$ Thus, a largescale prospective trial is needed to investigate whether tumor invasion to the arteries feeding the gallbladder is a risk factor.

There were several limitations to the present study. First, it was a single-center retrospective study and there might have been some bias in patient selection. Second, it was difficult to measure the AF. Thus, an AF unknown for the majority of SEMS was used in the present investigation, and consequently, we could not investigate the risk factors associated with different AFs. Nakai et al. ${ }^{10}$ suggested that low AF SEMS might reduce the incidence of cholecystitis; thus, in the future, we should perform a prospective study using low AF SEMS. Third, the flow of the contrast agent into the gallbladder was determined visually on fluoroscopic images, which is a non-quantitative method; thus, it was not possible to determine the relationship between the specific amount of bile containing contrast agent that entered the gallbladder and the onset of cholecystitis. Fourth, it is expected that the causes of early and late cholecystitis may differ. Ainley et al. ${ }^{21}$ suggested that early cholecystitis may be due to the occlusion of the OCD by the stent, while late cholecystitis may be due to tumor growth, causing cystic duct compression. Thus, if the proportion of the contrast agent injected into the gallbladder and cystic duct tumor invasion differs between the early and late cholecystitis groups, this may provide a suitable explanation for the mechanism involved in cholecystitis. However, the proportion of the contrast agent injected into the gallbladder and cystic duct tumor invasion did not differ between the early and late cholecystitis groups in this study. Therefore, further investigation is needed in a larger sample population.

In conclusion, the present study suggested that the contrast agent flow into the gallbladder and the tumor involvement to the OCD were risk factors for cholecystitis development following CSEMS placement. A prospective investigation is warranted to validate the effectiveness of EUS-BD for the prevention of cholecystitis in patients with OCD tumor involvement. 
Conflicts of Interest

The authors have no potential conflicts of interest.

Funding

None.

Author Contributions

Conceptualization: Masafumi Watanabe, Kosuke Okuwaki

Data curation: MW, KO, Jun Woo, Mitsuhiro Kida, Hiroshi Imaizumi, Tomohisa Iwai

Formal analysis: MW, KO

Investigation: $\mathrm{MW}, \mathrm{KO}$

Methodology: MW, KO

Project administration: MW, KO

Resources: MW, KO

Software: MW, KO

Supervision: MW, KO

Validation: MW, KO

Visualization: MW, KO

Writing-original draft: MW, KO

Writing-review\&editing: KO, JW, MK, HI, TI, Hiroshi Yamauchi, Toru Kaneko, Rikiya Hasegawa, Takahiro Kurosu, Naoki Minato, Hiroki Haradome, Wasaburo Koizumi

\section{ORCID}

Masafumi Watanabe:

Kosuke Okuwaki:

Jun Woo:

Mitsuhiro Kida:

Hiroshi Imaizumi:

Tomohisa Iwai:

Hiroshi Yamauchi:

Toru Kaneko:

Rikiya Hasegawa:

Naoki Minato:

Hiroki Haradome:

Wasaburo Koizumi:
Takahiro Kurosu: https://orcid.org/0000-0003-1278-9021 https://orcid.org/0000-0002-3671-1299 https://orcid.org/0000-0001-6564-6660 https://orcid.org/0000-0002-5794-1130 https://orcid.org/0000-0002-0103-614X https://orcid.org/0000-0001-6656-4499 https://orcid.org/0000-0002-4906-5601 https://orcid.org/0000-0003-1924-1755 https://orcid.org/0000-0002-1080-5983 https://orcid.org/0000-0003-4639-4001 https://orcid.org/0000-0002-2422-8136 https://orcid.org/0000-0001-5690-3549 https://orcid.org/0000-0001-9972-1083

\section{REFERENCES}

1. Soehendra N, Reynders-Frederix V. Palliative bile duct drainage - a new endoscopic method of introducing a transpapillary drain. Endoscopy 1980;12:8-11.

2. Cotton PB. Duodenoscopic placement of biliary prostheses to relieve malignant obstructive jaundice. Br J Surg 1982;69:501-503.

3. ASGE Technology Assessment Committee, Pfau PR, Pleskow DK, et al. Pancreatic and biliary stents. Gastrointest Endosc 2013;77:319-327.

4. Leung JW. History of bile duct stenting: rigid prostheses. In: Kozarek R, Baron T, Song H-Y, eds. Self-expandable stents in the gastrointestinal tract. New York (NY): Springer; 2013. p. 15-31.

5. Almadi MA, Barkun A, Martel M. Plastic vs. self-expandable metal stents for palliation in malignant biliary obstruction: a series of meta-analyses. Am J Gastroenterol 2017;112:260-273.

6. Kitano M, Yamashita Y, Tanaka K, et al. Covered self-expandable metal stents with an anti-migration system improve patency duration without increased complications compared with uncovered stents for distal biliary obstruction caused by pancreatic carcinoma: a randomized multicenter trial. Am J Gastroenterol 2013;108:1713-1722.

7. Saleem A, Leggett CL, Murad MH, Baron TH. Meta-analysis of randomized trials comparing the patency of covered and uncovered self-expandable metal stents for palliation of distal malignant bile duct obstruction. Gastrointest Endosc 2011;74:321-327.e1-e3.

8. Sogabe $\mathrm{Y}$, Kodama $\mathrm{Y}$, Honjo H, et al. Tumor invasion to the arteries feeding the gallbladder as a novel risk factor for cholecystitis after metallic stent placement in distal malignant biliary obstruction. Dig Endosc 2018;30:380-387.

9. Shimizu S, Naitoh I, Nakazawa T, et al. Predictive factors for pancreatitis and cholecystitis in endoscopic covered metal stenting for distal malignant biliary obstruction. J Gastroenterol Hepatol 2013;28:68-72.

10. Nakai $\mathrm{Y}$, Isayama $\mathrm{H}$, Kawakubo $\mathrm{K}$, et al. Metallic stent with high axial force as a risk factor for cholecystitis in distal malignant biliary obstruction. J Gastroenterol Hepatol 2014;29:1557-1562.

11. Suk KT, Kim HS, Kim JW, et al. Risk factors for cholecystitis after metal stent placement in malignant biliary obstruction. Gastrointest Endosc 2006;64:522-529.

12. Isayama H, Kawabe T, Nakai $Y$, et al. Cholecystitis after metallic stent placement in patients with malignant distal biliary obstruction. Clin Gastroenterol Hepatol 2006;4:1148-1153.

13. Nakai $Y$, Isayama $H$, Tsujino $T$, et al. Intraductal US in the assessment of tumor involvement to the orifice of the cystic duct by malignant biliary obstruction. Gastrointest Endosc 2008;68:78-83.

14. Isayama H, Hamada T, Yasuda I, et al. TOKYO criteria 2014 for transpapillary biliary stenting. Dig Endosc 2015;27:259-264.

15. Isayama $\mathrm{H}$, Nakai $Y$, Toyokawa $Y$, et al. Measurement of radial and axial forces of biliary self-expandable metallic stents. Gastrointest Endosc 2009;70:37-44.

16. Isayama H, Nakai Y, Hamada T, Matsubara S, Kogure H, Koike K. Understanding the mechanical forces of self-expandable metal stents in the biliary ducts. Curr Gastroenterol Rep 2016;18:64.

17. Li DF, Zhou CH, Wang LS, Yao J, Zou DW. Is ERCP-BD or EUS-BD the preferred decompression modality for malignant distal biliary obstruction? A meta-analysis of randomized controlled trials. Rev Esp Enferm Dig 2019;111:953-960.

18. Kim GH, Ryoo SK, Park JK, et al. Risk factors for pancreatitis and cholecystitis after endoscopic biliary stenting in patients with malignant extrahepatic bile duct obstruction. Clin Endosc 2019;52:598-605.

19. Klimczak T, Kaczka K, Klimczak J, Tyczkowska-Sieroń E, Tyczkowska A. Primary bacterial culture of bile and pancreatic juice in tumor related jaundice (TROJ) - is ascending cholangitis always our fault? Scand J Gastroenterol 2018;53:1569-1574.

20. Gregg JA, De Girolami P, Carr-Locke DL. Effects of sphincteroplasty and endoscopic sphincterotomy on the bacteriologic characteristics of the common bile duct. Am J Surg 1985;149:668-671.

21. Ainley CC, Williams SJ, Smith AC, Hatfield AR, Russell RC, Lees WR. Gallbladder sepsis after stent insertion for bile duct obstruction: management by percutaneous cholecystostomy. Br J Surg 1991;78:961-963. 